\title{
L'ASSM prend part à trois études scientifiques
}

Académie Suisse des Sciences Médicales (ASSM)

Correspondance:

Académie Suisse des Sciences

Médicales (ASSM)

Petersplatz 13

CH-4051 Bâle

mail[at]samw.ch
La conception de la fin de vie suscite un intérêt toujours croissant au sein de l'opinion publique. Le renforcement de l'autonomie, grâce aux instruments de prévoyance individuelle [1] introduits par le nouveau droit de protection de l'enfant et de l'adulte, mais également les différents débats sur le sens et la durabilité des traitements médicaux influencent les attentes des patients et de leurs proches. La réflexion sur le rôle et la responsabilité du corps médical dans l'accompagnement des patients en fin de vie fait depuis toujours partie des questions fondamentales relatives à la perception de l'activité médicale. Dans plusieurs de ses directives médico-éthiques, l'Académie Suisse des Sciences Médicales (ASSM) s'est exprimée sur les décisions difficiles qui se posent en fin de vie et s'est engagée depuis longtemps pour la reconnaissance et l'implémentation des soins palliatifs.

\section{Etude 1:}

Attitude du corps médical face

à l'assistance au suicide

Dans ses directives sur la prise en charge des patientes et patients en fin de vie [2] et dans sa prise de position «Problèmes de l'assistance médicale au suicide»[3], l'ASSM s'est exprimée sur la participation des médecins à l'assistance au suicide. Les directives n'excluent pas l'assistance au suicide par un médecin, mais la limite aux patients en fin de vie. Cette prise de position a déclenché des débats animés; d'aucuns ont même exigé une adaptation des directives [4]. En novembre 2012, le Bulletin des médecins suisses avait organisé une table ronde «Suizidhilfe - (k)eine ärztliche Aufgabe?» (Assistance au suicide - est-elle (ou non) une activité médicale?) [5].

Dans le contexte de ces débats, l'ASSM a décidé de mettre au concours une étude, dont le but est d'analyser avec précision l'attitude du corps médical face à l'assistance au suicide. Les résultats de cette étude serviront de base à une discussion qui se déroulera au niveau interne dans un premier temps et qui sera ensuite publiée.

En 2011, le Fonds National Suisse a lancé un programme national de recherche «Fin de vie» (PNR 67) [6]. Son but est de «produire de manière scientifique des connaissances servant à définir des orientations ou des actions dans le domaine de la dernière phase de la vie et de les mettre à la disposition des décideuses et décideurs du système de santé, de la politique et des professions qui s'occupent de la prise en charge des personnes en fin de vie». L'ASSM salue expressément cette initiative et participe activement à deux projets (étude 2 et étude 3 ).

\section{Etude 1: \\ Etude de l'ASSM sur l'attitude du corps médical face à l'assistance \\ au suicide \\ L'étude devra éclaircir les points suivants:}

- Attitude des médecins face à l'assistance au suicide, sur le fond et en rapport avec un groupe de patients déterminé.

- Attitude des médecins concernant le rôle juste du corps médical en matière d'assistance au suicide.

- Disposition des médecins à participer à un suicide assisté.

- Expériences des médecins en matière de suicide médicalement assisté.

\section{Etude 2:}

Décisions médicales en fin de vie:

\section{fréquence et tendances en Suisse}

De nos jours, nombreux sont ceux qui décèdent à un âge avancé et les décisions médicales (renoncement et/ou retrait thérapeutique) influencent ce processus. En 2001, des données détaillées sur la pratique médicale ont été relevées pour la première fois dans le cadre de l'étude «Medical End-of-life Decisions in Six European Countries (EURELD-Studie)» à laquelle a également participé la Suisse alémanique [7]. L'étude actuelle - réalisée cette fois dans toute la Suisse - permet d'avoir un aperçu de l'évolution de la pratique médicale et, le cas échéant, de savoir si - et en quoi la Suisse romande et la Suisse italienne se distinguent de la Suisse alémanique. L'ASSM avait déjà participé à la première étude en tant qu'«administratrice» et assumera également ce rôle dans la nouvelle étude. Les questionnaires remplis par les médecins seront adressés à l'ASSM, puis transmis au centre d'étude après avoir été anonymisés.

\section{Etude 3:}

L'incapacité de discernement en fin de vie et son évaluation

L'ASSM soutient un deuxième projet réalisé dans le cadre du PNR 67: l'étude «L'incapacité de discernement en fin de vie et son évaluation». La capacité ou 
Etude 2:

«Décisions médicales en fin de vie: fréquence et tendances en Suisse»

Prof. Dr Felix Gutzwiller, Dr Matthias Bopp, PD Dr Georg Bosshard, Dr Karin Faisst, Prof. Dr Samia Hurst, Dr Christoph Junker, Dr Margareta Schmid, Ueli Zellweger MSC

Le projet entend fournir des informations valables et actuelles sur la nature et la fréquence des décisions médicales en fin de vie en Suisse. Ce faisant, des comparaisons sont effectuées entre les régions linguistiques de Suisse, mais aussi avec d'autres pays européens. De plus, la comparaison avec la première étude de ce type réalisée en Suisse, permettra d'obtenir un aperçu de l'évolution dans le temps. 9000 certificats de décès de Suisse alémanique, de Suisse romande et du Tessin, sélectionnés de manière aléatoire, constituent le point de départ de cette étude. A partir du mois d'août 2013, les médecins ayant établi ces certificats de décès seront interrogés: quels actes médicaux ont été effectués avant le décès du patient et dans quelle intention, comment les processus décisionnels se sont-ils déroulés, et quelles étaient les personnes impliquées? Une information spécifique à l'intention des médecins paraîtra dans le Bulletin des médecins suisses $n^{\circ} 33$ du 14 août 2013 ainsi que dans PrimaryCare et la Revue médicale suisse dès le début de l'enquête.

l'incapacité de discernement a des conséquences importantes pour l'implication du patient dans les décisions thérapeutiques. Toutefois il n'est pas toujours aisé de transposer des concepts juridiques à des affaires médico-psychologiques et inversement. L'ASSM souscrit à ce projet, qui permet d'évaluer la capacité de discernement avec des standards fondés et clairement définis. Dans le cadre de cette étude, l'ASSM a participé à l'élaboration du questionnaire qui sera soumis aux médecins.

\section{Références}

1 Mandat pour cause d'inaptitude et directives anticipées.

2 Prise en charge des patientes et patients en fin de vie. Directives médico-éthiques. www.samw.ch/fr/ Ethique/Fin_de_vie.html
Etude 3:

«L'incapacité de discernement en fin de vie et son évaluation»

Prof. Dr Nikola Biller-Andorno, Dr Manuel Trachsel

Le but de ce projet est de contribuer à la définition de standards nationaux pour l'évaluation de la capacité de discernement. La dernière phase de la vie d'un être humain s'accompagne souvent d'un état d'incapacité de discernement. Or, en fin de vie, il se présente souvent des situations où des décisions complexes, requérant la capacité de discernement, doivent être prises. II faut donc que les médecins puissent vérifier si la patiente ou le patient est capable de discernement. S'il apparaît que la personne n'est pas (ou n'est plus) capable de discernement par rapport à une décision particulière, cela a des conséquences sur la marche à suivre, par exemple, sur l'entrée en vigueur de directives anticipées ou la mise en place d'une personne habilitée à représenter le patient. Sur la base de cette enquête auprès de 300 médecins, une étude empirique sur la pratique d'évaluation actuelle en Suisse sera réalisée.

3 Problèmes de l'assistance médicale au suicide - Prise de position de la Commission Centrale d'Ethique. www.samw.ch/fr/Ethique/Fin_de_vie.html

4 Schafroth M. Suizidhilfe ist Teil der ärztlichen Aufgaben - die Zeit ist reif für eine Anpassung der SAMW-Richtlinien. Bull Méd Suisses. 2012;93(34):1226-7.

5 Résumé de la table ronde du Bulletin des médecins suisses du 27 novembre 2012 à Bâle. Sax A. Suizidhilfe - (k)eine ärztliche Aufgabe? Bull Méd Suisses. 2013;94(4):108-11.

6 www.nfp67.ch/F/Pages/home.aspx

7 Van der Heide A, Deliens L, Faisst K, Nilstun T, Norup M, Paci E. End-of-life decision-making in 6 European Countries. Descriptive Study. Lancet. 2003;362:345-50.

\section{Articles interactifs}

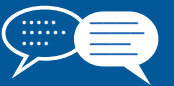

Vous souhaitez commenter cet article? II vous suffit pour cela d'utiliser la fonction «Ajouter un commentaire» dans la version en ligne. Vous pouvez également consulter les remarques de vos confrères sous: www.bullmed.ch/ numero-actuel/articles-interactifs/ 